\title{
Bilateral changes in cell density within the central auditory pathway upon chronic unilateral intra-cochlear stimulation
}

\author{
Basta $D^{1 *}$, Jansen $S^{2}$, Groschel $M^{1}$, Schwitzer $S^{1}$, Boyle $P^{3}$, Ernst $A^{1}$ and Seidl $R^{1}$ \\ ${ }^{1}$ Department of Otolaryngology at UKB, University of Berlin, Germany \\ ${ }^{2}$ Department of Biology, Humboldt-University of Berlin, Germany \\ ${ }^{3}$ Advanced Bionics GmbH, Hanover, Germany
}

\begin{abstract}
Cochlear implants have been applied successfully for the treatment of unilateral hearing loss with quite a surprising benefit. One reason for this successful treatment could be the occurrence of neuroplastic changes within the central auditory pathway upon a bimodal stimulation. Important parameters of the electro-stimulation which could trigger neuroplastic changes are largely unknown. The present study, therefore, investigated at a cellular level, the effect of different stimulation rates and intensities on key structures of the central auditory pathway.

Normal-hearing guinea pigs were mechanically single-sided deafened through a standard HiFocus1j electrode array being inserted into the first turn of the cochlea. Four to five electrode contacts were available for stimulation.

After eCAP-threshold based speech processor fitting, three experimental groups were stimulated 16 hours per day for 90 days. A HiRes ${ }^{\otimes}$-strategy, based on one of three stimulation rates, low-rate $275 \mathrm{pps} / \mathrm{ch}$, mid-rate $1500 \mathrm{pps} / \mathrm{ch}$, or high-rate $5000 \mathrm{pps} / \mathrm{ch}$ was used, with the animals living in a standardised free field auditory environment. Afterwards, the cell density was determined in key structures of the auditory pathway. Results were compared to those of unilateral implanted but not stimulated controls.

A bilateral conservation of all the brain structures investigated was found in the low- and high-rate groups. A significant cell loss was observed in the mid-rate group. This group also showed the highest mean stimulation current. Unilateral intra-cochlear electrical-stimulation leads to bilateral central nervous changes which correlate with the stimulation current applied rather than the stimulation rate applied.
\end{abstract}

\section{Introduction}

Unilateral hearing loss produces impaired spatial hearing and reduces speech understanding in noise, leading to a reduction in quality of life. Various studies [1-3] have shown that unilateral hearing loss is accompanied by a variety of changes within the peripheral and central auditory pathways. Besides the well documented degeneration of peripheral structures, changes within the central auditory pathway have also been investigated recently. Some studies report on modulation of network activity within the cochlear nucleus $(\mathrm{CN})$, the inferior colliculus (IC), and the auditory cortex (AC). Deafness related changes typically mean a decrease in inhibition and an increase in spontaneous or evoked activity [4-10]. Our group recently showed that changes in neural activity can be normalized by intra-cochlear electrostimulation [1].

As a result of deafferentation, structural modifications within the central auditory pathway are highly probable following unilateral hearing loss. Demyelination and a degeneration of the spiral ganglion cell (SGC) population is triggered by hair cell loss $[11,12]$. This can lead in turn to a damage of the $\mathrm{CN}$, followed by a partial degeneration of the entire auditory pathway. The damage becomes apparent as a cell loss, as shown in several previous studies $[11,13,14]$.

Nowadays, unilateral hearing loss is being successfully treated with cochlear implantation $[9,10]$. Interestingly, electrical stimulation arrests the progression of degeneration within the spiral ganglion and the central auditory pathway. The conservation of central structures is possible through early and adequate intra-cochlear electrical stimulation [16]. One prominent effect is the reduction of structural changes within the DCN $[13,15]$.

However, it is largely unknown which stimulation parameters of cochlear implants are most advantageous in supporting the conservation of auditory structures. This question is of utmost importance for unilateral cochlear implant recipients, since structural degeneration could influence the bimodal processing in a negative manner. One potentially important parameter is the stimulation rate, since this parameter influences the firing rate of the auditory nerve and thus determines the input strength for central auditory processing.

The present study, therefore, aims to investigate the anatomical changes of key structures within the central auditory pathway (DCN, IC, medial geniculate body (MGB) and $\mathrm{AC}$ ) that are produced by chronic unilateral intra-cochlear electrical stimulation using different stimulation rates.

${ }^{\star}$ Correspondence to: Dietmar Basta, Department of Otolaryngology at ukb, Center for Applied Medical Technology, University of Berlin, Warener Str. 7, 12683 Berlin, Germany, E-mail: dietmar.basta@rz.hu-berlin.de

Key words: binaural conservation, cochlear Implant, unilateral electrical stimulation, cell density, stimulation rate, stimulation intensity

Received: October 31, 2019; Accepted: November 12, 2019; Published: November 15, 2019 


\section{Materials and methods}

The experimental protocol was approved by the governmental commission for animal studies (LaGeSo Berlin, Germany; PI: Dr. Dietmar Basta, approval No. G0280/04, G0392/08 and G0417/10). Experiments were carried out in accordance with the EU-Directive $2010 / 63 / \mathrm{EU}$ on the protection of animals used for scientific purposes. All efforts were made to minimize pain or discomfort.

\section{Cochlear implant surgery}

Sixteen adult guinea pigs (Cavia porcellus; Dunkin Hartley), verified as normal hearing by frequency specific auditory brainstem response measures, were unilaterally implanted under anaesthesia (Ketamine/ Xylazine). The cochlear implant was a HiRes $90 \mathrm{k}^{\circledR}$ device having 16 intra-cochlear electrode contacts available on its HiFocus $1 j^{\circledR}$ electrode array (Advanced Bionics, Valencia, California, USA). Typically, the first 4-5 electrode contacts were inserted into the cochlea and hence could be used to stimulate the cochlear nerve fibres within the first turn. The animals were deafened mechanically by the insertion of a human-sized cochlear implant electrode array producing a ruputure of the basilar membrane and hence mixing endolymph and perilymph within the cochlea.

The proper insertion and function of the implant was confirmed intraoperatively. Both electrode contact impedances and amplitude growth functions for the electrically evoked compound action potentials (eCAPs) were measured for all inserted electrode contacts.

The same method of implantation was used for the control animals.

\section{Frequency specific measurement of auditory brainstem response thresholds}

Frequency-specific auditory brainstem response thresholds at 2,4 , $6,8,10,12,14,16,18$, and $20 \mathrm{kHz}$ were determined under anaesthesia (Ketamine/Xylazine), before, six weeks after surgery and at the end of the experiment, following 90 days of electrical stimulation.

Sub-dermal needle electrodes were placed at the vertex (reference), mastoid (active), and at one foot (ground) while tone-burst stimuli were delivered monaurally with in-ear tip transducers at different SPLs from a sine-wave generator (Modell SSU2, Werk für Fernmeldewesen, Berlin, Germany). A broadband noise with a peak level of $50 \mathrm{~dB}$ SPL was delivered to mask the hearing contra-lateral ear during measurement of the implanted ear. Recordings were carried out with a Viking IV measurement system (Viasys Healthcare, Conshohocken, Pennsylvania, USA). The brainstem responses (recording time 10 $\mathrm{ms}$ after stimulus presentation) were amplified $(100,000 \mathrm{x})$, filtered (bandpass $0.15-3 \mathrm{kHz}$ ), and averaged (300x) by the Viking IV. A linear regression line was fitted to the linear part of the relationship between wave IV/V-amplitude and stimulus level (in $\mathrm{dB}$ SPL) for each frequency tested. The equation of this linear function was used to determine the auditory threshold, that is, the stimulus level at which zero IV/V amplitude would occur.

\section{Sound processor fitting}

Six weeks after surgery, a sound processor (Auria ${ }^{\circledR}$, Advanced Bionics, Valencia, California, USA) was mounted on the back of each animal in the experimental groups and programmed with M-levels (upper limit of the electrical stimulation) based on the threshold of neural response imaging (tNRI), the eCAP threshold. The lower limit of the electrical stimulation (T-level) were set to $10 \%$ of M-levels. This tNRI-based sound processor fitting is commonly used in children, or in noncompliant adult implant recipients.

All non-used channels of the sound processor had their outputs set to zero to avoid changes in the frequency filter bands (frequency range per channel), which would occur, through elimination of channels.

Finally, the loudness was adapted to the Preyer-reflex threshold by changing the overall profile of the stimulation program. The Preyer reflex is a startle response involving pricking back of the ears upon a sudden loud sound produced by the activation of the sound processor in a noisy environment. The overall profile of the stimulation program was decreased or increased until a first Preyer response was detected.

\section{Intra-cochlear electrical stimulation}

The intra-cochlear electrical stimulation was delivered to the experimental groups via a cochlear implant that supported a stimulation rates of $275 \mathrm{pps} / \mathrm{ch}$ (pulse width $227.2 \mu \mathrm{s}$ per phase) for the "low stimulation rate" group (LSR; $n=3$ ), a stimulation rate of 1513 pps/ ch $(41.1 \mu$ s per phase) for the "medium stimulation rate" group (MSR; $\mathrm{n}=3)$ and a stimulation rate of $5156 \mathrm{pps} / \mathrm{ch}(10.8 \mu$ s per phase) for the "high stimulation rate" group (HSR; $n=4)$. All experimental groups were compared to an implanted but not stimulated control group (controls; $\mathrm{n}=6$ ).

The HiRes-sound coding strategy running on the Auria ${ }^{\circledR}$ audio processor was used to deliver the stimulation. The "Lord of the Rings" radio play [17] was provided at a level of $65 \mathrm{~dB}$ SPL as a standardized background acoustical environment. This radio play contains human voices as well as species-related environmental sounds (e.g., rustling of leaves and grass, wind blowing, cry of birds, cracking of perches). The power spectrum of the standardized acoustic signal is shown in Figure 1. The main power of the signal is related to frequencies that are within the filter bands of electrode contacts one to five (center frequency of electrodes: E1 $=333 \mathrm{~Hz}$; E2 $=455 \mathrm{~Hz}$; E3 $=540 \mathrm{~Hz}$; E4=642 Hz; E5=763 $\mathrm{Hz}$ ). The experimental group, as well as the controls, experienced similar acoustic environments for 16 hours per day. During this period, the sound processor was activated. In total, the experimental groups experienced 90 days of single-sided electrical stimulation via the cochlear implant.

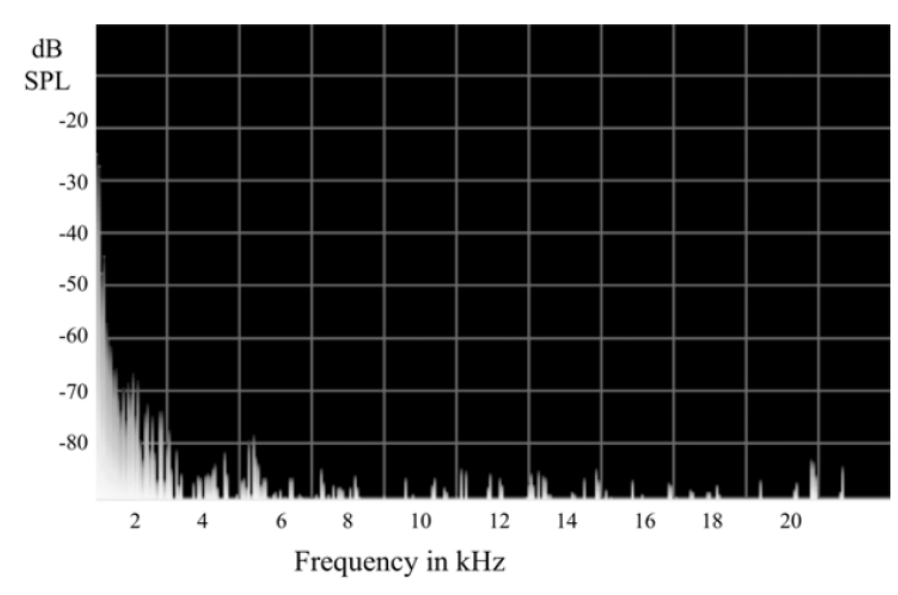

Figure 1. Power spectrum of the frequencies within the standardized auditory signal, which was applied to the guinea pigs for 90 days ( $16 \mathrm{~h}$ per day). Centre frequency of the inserted intra-cochlear electrodes are: E1 $=333 \mathrm{~Hz}, \mathrm{E} 2=455 \mathrm{~Hz}, \mathrm{E} 3=540 \mathrm{~Hz}, \mathrm{E} 4=642 \mathrm{~Hz}, \mathrm{E} 5=763 \mathrm{~Hz}$ 


\section{Histology}

At the end of the experiment, under anaesthesia (Ketamine/ Xylazine), the animals were perfused and fixated through the left ventricle with $\mathrm{NaCl} 0,9 \%(20 \mathrm{ml} / \mathrm{min}, 0.1 \mathrm{bar})$ followed by $4 \%$ paraformaldehyde in buffered saline $(0.2 \mathrm{M})$. The brain was removed and stored in $4 \%$ paraformaldehyde $\left(24 \mathrm{~h}\right.$ at $\left.7^{\circ} \mathrm{C}\right)$. The tissue was embedded in paraffin by using an automated embedder (Leica EG 1160 Histoembedder, Leica Microsystems, Wetzlar, Germany) and cut (10 $\mu \mathrm{m}$ thick slices) with a manual rotary microtome (Leica Microsystems, Wetzlar, Germany). The slices were stained with the standard hematoxylin and eosin staining method. Microphotographs of the areas of interest were taken with a Canon EOS 1000D camera via a microscope (Axiovert 25C, Carl Zeiss, Germany).

The cell density (cells per $\mathrm{mm}^{2}$ ) was determined in a defined area within the DCN, the IC, the MGB and the AC. In the DCN the cell density was determined separately for the three layers (molecular layer, intermediate layer, deep layer). In the IC and MGB the cell density was determined only in the central region, whereas all six layers of the $\mathrm{AC}$ were examined.

\section{Statistical procedures}

The histological data of the experimental groups and the controls were analysed by comparing the cell densities obtained from the implanted and non-implanted sides ( $\mathrm{t}$-/u-test respectively, depending on data distribution). The significance level for all statistical tests was $\mathrm{p}<0.05$. A Bonferroni alpha-correction was applied for multiple comparisons.

\section{Results}

\section{Frequency-specific auditory Brainstem Response Threshold} (ABR)

All animals showed normal ABR thresholds preoperatively according to published values $[18,19]$. Six weeks after surgery there were no detectable response on the implanted side upon auditory stimulation up to $100 \mathrm{~dB}$ SPL. The non-implanted side showed no significant threshold shift when compared to the controls after 90 days of electrical stimulation.

\section{Cell density}

Cochlear nucleus: The cell density was determined for the molecular layer (layer 1), the intermediate layer (layer 2) and the deep layer (layer 3) of the DCN. Results of the treatment groups were compared with those of the controls (LSR, $\mathrm{n}=705$ slices [control, $\mathrm{n}=357$ slices]; MSR, $\mathrm{n}=540$ slices [control, $\mathrm{n}=351$ slices]; HSR, $\mathrm{n}=297$ slices [control, $n=360$ slices]). Electrically stimulated animals of the LSRgroup showed a significant bilateral reduction of cell loss in all DCNlayers compared to the controls (Figure 2). This reduction in cell loss was also observed in layer 3 of the stimulated side in the HSR-group. No stimulation effect was found in layers 1 and 2 for the HSR group. The MSR-group showed a significant cell loss in all three layers of the non-stimulated side and a bilateral cell loss in layer 2.

Inferior colliculus and medial geniculate body: No cell loss could be found bilaterally within the IC for any of the experimental groups compared to the controls (Figure 3) (LSR, n=120 slices [control, $\mathrm{n}=120$ slices]; MSR, $\mathrm{n}=120$ slices [control, $\mathrm{n}=120$ slices]; HSR, $\mathrm{n}=120$ slices [control, $\mathrm{n}=120$ slices]). A bilateral conservation was observed for the LSR-group and the MSR-group within the IC and a unilateral conservation for the HSR-group on the non-stimulated side.

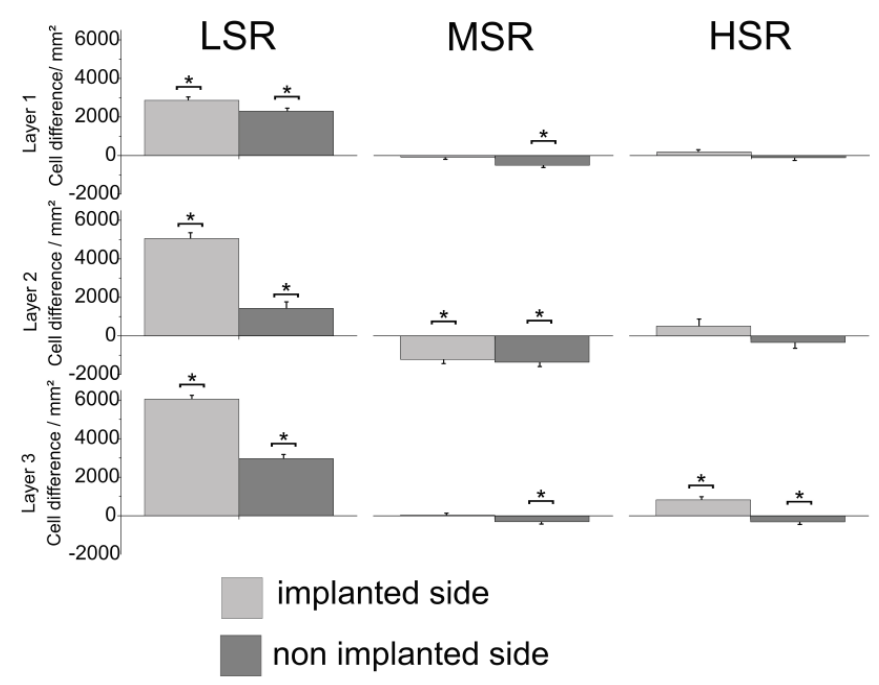

Figure 2. Mean cell density differences in the three layers of the dorsal cochlear nucleus (DCN) compared to the same area of implanted but not stimulated controls. Asterisks indicate significant differences. $L S R=$ low stimulation rate group; $M S R=$ medium stimulation rate group; $\mathrm{HSR}=$ high stimulation rate group

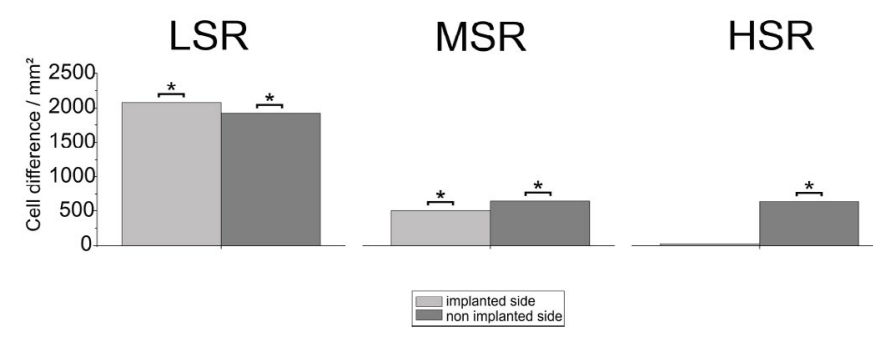

Figure 3. Mean cell density differences in the inferior colliculus (IC) compared to the same area of implanted but not stimulated controls. Asterisks indicate significant differences. $\mathrm{LSR}=$ low stimulation rate group; $\mathrm{MSR}=$ medium stimulation rate group; $\mathrm{HSR}=$ high stimulation rate group

Bilateral conservation within the MGB was also observed for the LSR-group and the HSR-group (LSR, $\mathrm{n}=120$ slices [control, $\mathrm{n}=120$ slices]; HSR, $\mathrm{n}=120$ slices [control, $\mathrm{n}=120$ slices]). The MSR-group (MSR, $\mathrm{n}=120$ slices [control, $\mathrm{n}=119$ slices]) showed a significant cell loss on the non-stimulated side (Figure 4).

Primary auditory cortex : Figure 5 shows the average cell density in the AC compared to the controls (LSR, $n=432$ slices [control, $n=575$ slices]; MSR, $n=714$ slices [control, $n=575$ slices]; HSR, $n=546$ slices [control, $\mathrm{n}=573$ slices]). Bilateral conservation of neuronal structure compared to the controls, was found in all layers of the LSR-group. Such a bilateral conservation could be observed only in layer 3 of the HSRgroup. All other layers of the HSR-group showed no effect bilaterally. A significant bilateral cell loss was detected in layers 1, 3 and 5 of the MSR-group, whereas layers 2 and 6 showed a cell loss only on the nonstimulated side.

\section{Discussion}

Data from the present study shows different effects of unilateral intra-cochlear electrical stimulation for the three different stimulation rates on four major structures of the central auditory pathway (DCN, IC, MGB and AC) in single-sided deaf animals.

\section{Bilateral effects}

This study reveals that the ascending auditory pathway is affected bilaterally by chronic intra-cochlear electrical stimulation. This 


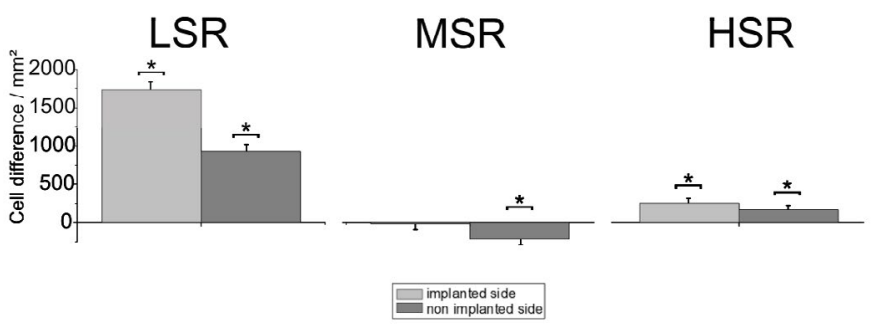

Figure 4. Mean cell density differences in the medial geniculate body (MGB) compared to the same area of implanted but not stimulated controls. Asterisks indicate significant differences. $\mathrm{LSR}=$ low stimulation rate group; $\mathrm{MSR}=$ medium stimulation rate group; $\mathrm{HSR}=$ high stimulation rate group
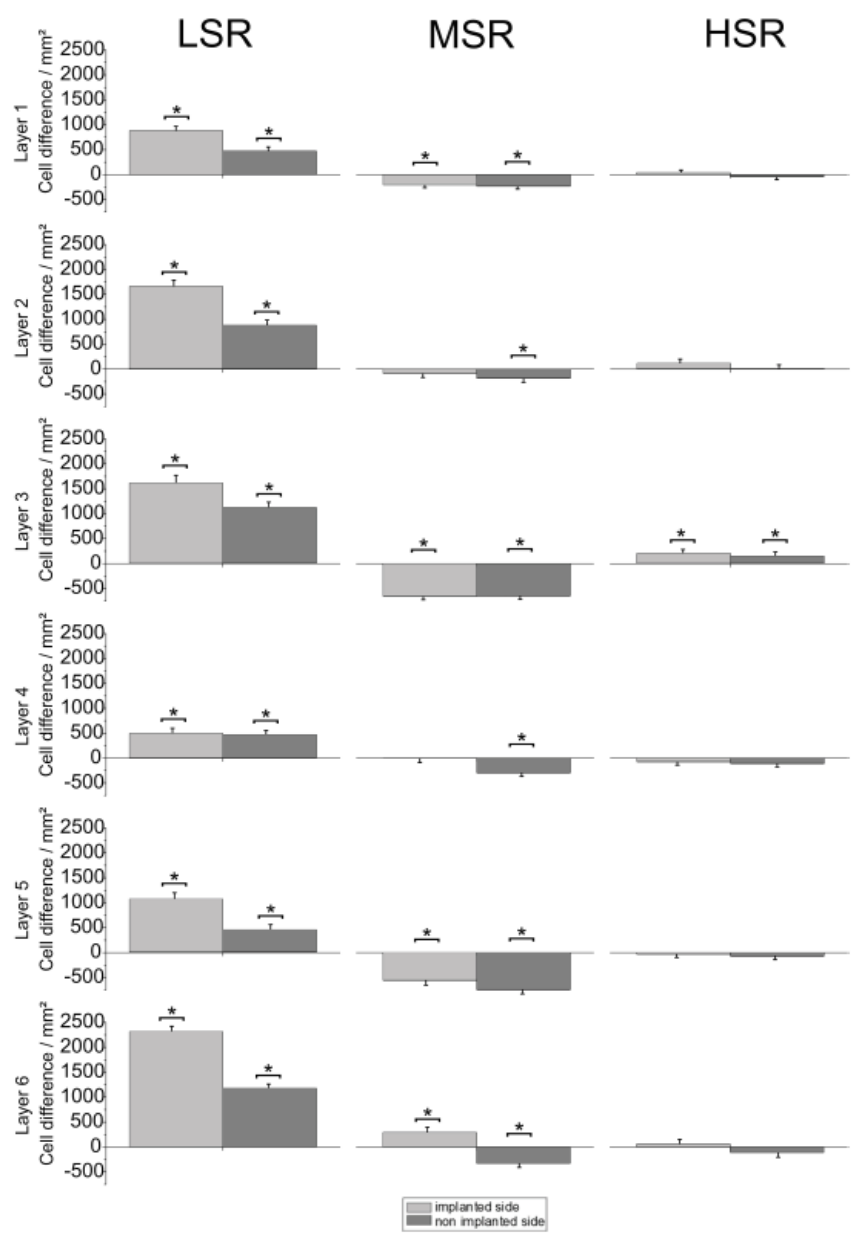

Figure 5. Mean cell density for the six layers of the auditory cortex compared to the same areas of implanted but not stimulated controls. Asterisks indicate significant differences. $\mathrm{LSR}=$ low stimulation rate group; $\mathrm{MSR}=$ medium stimulation rate group; HSR=high stimulation rate group

interesting finding could be based on the interrelation between inputs of both ears within the structures investigated.

One half of the ipsilateral neurons in the ventral cochlear nucleus (VCN) [20], as well as neurons in the DCN [21,22], are excited by contra-lateral acoustic stimulation. A missing input from the cochlea leads to a reduction in suppression for the cochlear nucleus as well as the IC $[6,8,23]$.

The IC receives diverse afferents from the ipsilateral and contralateral VCN, DCN, SOC and the contralateral IC. These inputs inhibit deprivation of the IC and result in a bilateral conservation [2426]. The MGB receives direct input from the ipsilateral IC, the nucleus reticularis [27,28], as well as indirectly from the contralateral IC $[24,26]$. The MGB is missing a direct connection to the MGB of the other side. Nonetheless, a sensitivity for binaural stimuli has been shown $[28,29]$.

The input from the subcortical structures occur in multiple AC layers. There are inputs from the ipsilateral ventral (layers $3+4$ ) and medial MGB (layers 1+6) into the primary auditory cortex, as well as from the contralateral AC (layer 3) [28,30,31].

The specific bilateral effects detected in the present study could be related to the, at least partly, bilateral bimodal input to the brain structures under study. During unilateral electrical stimulation with a $\mathrm{CI}$ in presence of a normal hearing contra-lateral ear, both pathways (ipsi- and contralateral) were stimulated (electrically and acoustically) simultaneously. Related to this is different signal coding and processing within the entire pathway. Neuroplastic adaptive changes, as described in this study, are required for the rehabilitation of different hearing cues in unilateral cochlear implantees. A previous study showed that one feature that is obviously processed differently, the interaural time difference, was compensated by neuroplastic changes within the rehabilitation period [32].

\section{Specific structural changes}

Earlier studies have shown that sensory deprivation by a cochlear lesion led to a degeneration of the DCN $[11,33,34]$. In the present study, a statistically significantly higher cell density was observed in the DCN. This was observed bilaterally for the LSR-group and on the stimulated side for the HSR-group (compared to a unilaterally implanted but not stimulated control). These results support findings which show a conservation of neuronal structures upon electrical stimulation $[11,13,35]$. Surprisingly, a bilateral loss of DCN neurons was found in the MSR-group. However, these results do not correlate in all three DCN layers with the stimulation rates.

This also holds true for the MGB and all layers of the AC. Both, the LSR-group and the HSR-group showed mainly bilateral structural conservation whereas a cell loss was observed in the MSR-group. Thus, a factor independent of the stimulation rate should be responsible for the observed decline in cell density. Since all surgeries were carried out under similar conditions (e. g. surgeon, anesthetics, gender, age, approach), the speech processor fitting could be responsible for the different results. The fitting procedure has a subjective component even in human patients. The audiologist increases stimulation current until the subjective most comfortable loudness is achieved. Loudness was adjusted in the present study by the visual detection of the Preyer-reflex. This procedure could possibly be biased by the individual threshold detection. To exclude this potential bias we analyzed the stimulation intensity across the stimulation rate groups.

\section{Stimulation intensity}

The stimulation intensity values of the three groups correlate well with the cell densities in the DCN, MGB and the AC. The LSR and HSR groups with the lower mean stimulation intensities $(\mathrm{LSR}=86.25 \mathrm{CU}$ $[ \pm 5.9], \mathrm{HSR}=161.80 \mathrm{CU}[ \pm 5.4]$ ) showed conservational effects for cell density, whereas the MSR group, with significantly higher stimulation intensity (191.92 CU [ \pm 20.2$]$ ), showed a cell loss compared to the implanted but not stimulated controls (Figure 6). The significantly lower mean intensity of the LSR-group resulted in a bilateral conservation in all the nuclei. Following a medium stimulation intensity, structural conservation, or even no cell density loss, could be observed (HSRgroup). 


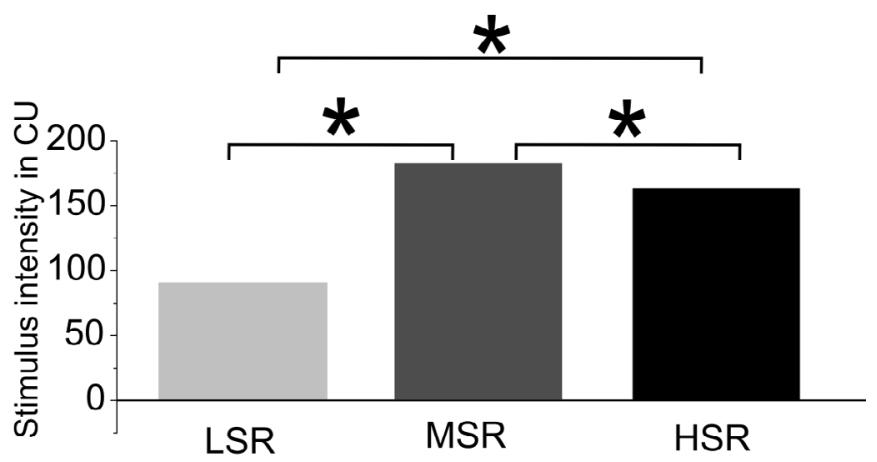

Figure 6. Stimulus intensity of the three experimental groups in clinical units (CU). $\mathrm{LSR}=$ low stimulation rate group; $\mathrm{MSR}=$ medium stimulation rate group; $\mathrm{HSR}=$ high stimulation rate group

One explanation for the dosage dependent effect of stimulation current on cell density is that the increased stimulation intensity enhances neuronal activity, which in turn induces necrotic or apoptotic processes in the lower auditory pathway. This happens on a fairly longterm basis through a strong calcium influx [36]. Thus, overstimulation in turn leads to an increased neurotransmitter release that can have a necrotic effect (glutamate excitotoxicity) [36].

A deprivation of higher auditory structures could be the consequence of the damaged auditory brainstem. Reduced excitatory input may result in a decreased cell density in the MGB and AC. Such effects were previously described after noise exposure [37]. However, excito-toxic effects appear to be also possible in higher auditory structures, since the IC, as the main source for afferent inputs to the MGB and thus the AC, showed a bilateral structural conservation in all treatment groups. Hence deprivation within the MGB and the AC is limited. The reason why the IC is more robust against overstimulation or deprivation is unknown. It could be related to the relay function of IC neurons which receive multiple inputs from the lower auditory pathway for an integrated processing of signal properties. This prevents IC neurons from total deprivation.

\section{Conclusion}

In essence, the results of the present study demonstrate that bilateral structural changes within the entire central auditory pathway are induced by a unilateral cochlear implant stimulation. The electrical input using biphasic, charge balanced pulses, prevents neuronal cell loss following deafness induced deprivation, regardless of the stimulation rate. High stimulation current seems to reverse the conservational effects of the electrical input. More research is required to find out safe current limits for chronic unilateral cochlear implant stimulation in single-sided deaf patients.

\section{Acknowledgements}

This study was supported by Advanced Bionics GmbH, Hanover, Germany.

\section{Conflicts of interest}

P.B. is employed in a scientific role by Advanced Bionics $\mathrm{GmbH}$, Hanover, Germany. All other authors disclose no conflicts of interest.

\section{References}

1. Basta D, Gotze R, Groschel M (2015) Bilateral changes of spontaneous activity within the central auditory pathway upon chronic unilateral intracochlear electrical stimulation. Otol Neurotol 36: 1759-1765.
2. Syka J (2002) Plastic changes in the central auditory system after hearing loss, restoration of function, and during learning. Physiol Rev 82: 601-636. [Crossref]

3. Moller AR (2006) Neural plasticity in tinnitus. Prog Brain Res 157: 365-372. [Crossref]

4. Kaltenbach JA (2000) Neurophysiologic mechanisms of tinnitus. J Am Acad Audiol 11: 125-137. [Crossref]

5. Kaltenbach JA, Afman CE (2000) Hyperactivity in the dorsal cochlear nucleus after intense sound exposure and its resemblance to tone-evoked activity: a physiological model for tinnitus. Hear Res 140: 165-172.

6. Mossop JE, Wilson MJ, Caspary DM (2000) Down-regulation of inhibition following unilateral deafening. Hear Res 147: 183-187.

7. Salvi RJ, Wang J, Ding D (2000) Auditory plasticity and hyperactivity following cochlear damage. Hear Res 147: 261-274. [Crossref]

8. Vale C, Juiz JM, Moore DR (2004) Unilateral cochlear ablation produces greater loss of inhibition in the contralateral inferior colliculus. Eur J Neurosci 20: 2133-2140.

9. Hassepass F, Aschendorff A, Wesarg T (2013) Unilateral deafness in children audiologic and subjective assessment of hearing ability after cochlear implantation Otol Neurotol 34: 53-60.

10. Távora-Vieira D, De Ceulaer G, Govaerts PJ (2014) Cochlear implantation improves localization ability in patients with unilateral deafness. Ear Hear 5: 1-6.

11. Hardie NA, Shepherd RK (1999) Sensorineural hearing loss during development: morphological and physiological response of the cochlea and auditory brainstem. Hear Res 128: 147-165. [Crossref]

12. Shepherd RK, Hardie NA (2001) Deafness-induced changes in the auditory pathway: implications for cochlear implants. Audiol Neurootol 6: 305-318.

13. Lustig LR, Leake PA, Snyder RL (1994) Changes in the cat cochlear nucleus following neonatal deafening and chronic intracochlear electrical stimulation. Hear Res 74: 2937.

14. Matsushima JI, Shepherd RK, Seldon HL (1991) Electrical stimulation of the auditory nerve in deaf kittens: effects on cochlear nucleus morphology. Hear Res 56: 133-142.

15. Matsushima JI, Shepherd RK, Seldon HL (1991) Electrical stimulation of the auditory nerve in deaf kittens: effects on cochlear nucleus morphology. Hear Res 56: 133-142.

16. Mitchell A, Miller JM, Finger PA (1997) Effects of chronic high-rate electrical stimulation on the cochlea and eighth nerve in the deafened guinea pig. Hear Res 105: 30-43.

17. Tolkien JRR, Steinbach P, Lau B (2003) Der Herr der Ringe Horspiel. Munchen: Der Horverl 4: 10 CDs.

18. Hood LJ, Berlin CI, Heffner RS (1991) Objective auditory threshold estimation using sine-wave derived responses. Hear Res 55: 109-116.

19. Ruggero MA, Temchin AN (2002) The roles of the external, middle, and inner ears in determining the bandwidth of hearing. Proc Natl Acad Sci U S A 99: 13206-13210. [Crossref]

20. Bledsoe SC, Koehler S, Tucci DL (2009) Ventral cochlear nucleus responses to contralateral sound are mediated by commissural and olivocochlear pathways. $J$ Neurophysiol 102: 886-900.

21. Brown MC, Drottar M, Benson TE, Darrow K (2013) Commissural axons of the mouse cochlear nucleus. J Comp Neurol 521: 1683-1696. [Crossref]

22. Mast TE (1970) Binaural interaction and contralateral inhibition in dorsal cochlear nucleus of the chinchilla. J Neurophysiol 33: 108-115.

23. McAlpine D, Martin RL, Mossop JE (1997) Response properties of neurons in the inferior colliculus of the monaurally deafened ferret to acoustic stimulation of the intact ear. J Neurophysiol 78: 767-779.

24. Knipper M, Van Dijk P, Nunes I (2013) Advances in the neurobiology of hearing disorders: recent developments regarding the basis of tinnitus and hyperacusis. Prog Neurobiol 111: 17-33.

25. Malmierca MSMMA (2004) Auditory system, In: Paxinos G, ed. The rat nervous system, The: Academic Press 5: 997-1006.

26. Møller AR (2006) Hearing: anatomy, physiology, and disorders of the auditory system Oxford: Academic.

27. Pinault D (2004) The thalamic reticular nucleus: structure, function and concept. Brain Res Brain Res Rev 46: 1-31. [Crossref] 
28. Webster DB, Popper AN, Fay RR (1992) The mammalian auditory pathway: Neuroanatomy: Springer-Verlag.

29. Aitkin LM (1973) Medial geniculate body of the cat: responses to tonal stimuli of neurons in medial division. J Neurophysiol 36: 275-283. [Crossref]

30. Code RA, Winer JA (1985) Commissural neurons in layer III of cat primary auditory cortex (AI): pyramidal and non-pyramidal cell input. J Comp Neurol 242: 485-510. [Crossref]

31. Winer JA, Sally SL, Larue DT (1999) Origins of medial geniculate body projections to physiologically defined zones of rat primary auditory cortex. Hear Res 130: 42-61.

32. Francart T, Brokx J, Wouters J (2009) Sensitivity to interaural time differences with combined cochlear implant and acoustic stimulation. J Assoc Res Otolaryngol 10: 13141.
33. Clark GM, Shepherd RK, Franz BKH (1988) The histopathology of the human temporal bone and auditory central nervous system following cochlear implantation in a patient: Correlation with psychophysics and speech perception results. Acta Otolaryngologica 105: 1-65.

34. Miller JM, Sutton D, Webster DB (1980) Brainstem histopathology following chronic scala tympani implantation in monkeys. Ann Otol Rhinol Laryngol 4: 89.

35. Powell TP, Erulkar SD (1962) Transneuronal cell degeneration in the auditory relay nuclei of the cat. $J$ Anat $96: 249-268$. [Crossref]

36. Salinska E, Danysz W, Lazarewicz JW (2005) The role of excitotoxicity in neurodegeneration. Folia neuropathologica/Association of Polish neuropathologists and medical research centre. Polish Academy of Sciences 43: 322-339.

37. Basta D, Tzschentke B, Ernst A (2005) Noise-induced cell death in the mouse medial geniculate body and primary auditory cortex. Neurosci Lett 381: 199-204. [Crossref]

Copyright: (02019 Basta D. This is an open-access article distributed under the terms of the Creative Commons Attribution License, which permits unrestricted use, distribution, and reproduction in any medium, provided the original author and source are credited. 